\title{
Can standardized and evidence based plant immunomodulator (rice bran arabinoxylan concentrate) increase the effect of Gemcitabine in a synergistic manner? Case report of a patient with ductal pancreas carcinoma
}

\author{
Tibor Hajto* \\ Medical University Pecs, Hungary
}

\begin{abstract}
Background: Pancreatic ductal carcinoma is leading cause of cancer mortality. Gemcitabine with nab-Paclitaxel as a standard first line therapy is reporting improved clinical parameters but they are only transient. Immunological observations suggest that in background of this rapid resistance a tumor induced dysregulation of immune balance can also play a role. As it was shown the tumor-induced type- 2 innate immune cells activate various inhibitory regulatory cells and growth factors which can diminish both the activity of immune effectors and the immune sensitivity of tumor cells. As it well known, the immune sensitivity related to the MHC class-I chain-related A / B (MICA and MICB) stress molecules on tumor cells which are the ligands of the most important killing activator receptor (NKG2D) on natural killer (NK) cells. Because of the discovery several years ago, that Gemcitabine can enhance the expression of MICA and MICB molecules on tumor cells, its combination with an evidence based immunomodulator has a growing clinical interest.
\end{abstract}

Material and methods: In this case report a patient with metastatic and inoperable pancreas ductal adenocarcinoma is presented who was treated with gemcitabine (1678 mg) and nab-paclitaxel (210 mg) on 1.- 8. -15. days of months given eight cycles. This therapy was regularly combined with $45 \mathrm{mg} / \mathrm{kg}$ Biobran /MGN-3 given per oral three times a week which is a standardized rice bran arabinoxylan concentrate.

Results: In a now 56 years old patient an inoperable ductal adenocarcinoma (39x46 mm) in caudal part of pancreas with multiplex liver metastases (10-30 mm) was established by CT and biopsy. He was in a nearly terminal stage (having $27 \mathrm{~kg}$ decrease in body weight and intensive pains). Three months after the start of the therapy a remission of pancreas tumor $(25 \times 38 \mathrm{~mm})$ and in liver metastases average 3-10 mm decreases were established by CT. Seven months later in pancreas a complete remission was found and his liver metastases showed further 3-6 mm decreases. After 10 months these remissions were also established. The patient has not now any complaints and is able to work $100 \%$.

Conclusion: The combination of gemcitabine with evidence based and standardized immunomodulators (such as MGN-3) may open new strategies in the tumor therapy.

\section{Introduction}

Pancreatic ductal adenocarcinoma (PDAC) constitutes $90 \%$ of pancreatic cancer and it is the fourth leading cause of cancer-related death in the world. In addition, PDAC belongs to one of the most chemo-resistant cancer. Most of the available treatments are palliative reducing the disease-related symptoms and prolonging the survival. Therapies with targeting cancer associated molecular pathways have also not given satisfactory results [1]. Growing evidences suggest that in the unfavorable prognosis of PDAC a tumor induced immune dysregulation can play an important role $[2,3]$.

Despite of the great technical development of tumor diagnostics, at the time of the first recognition of a malignant tumors which have usual a higher diameter than $5 \mathrm{~mm}$, the numbers of the cells are at least more millions. At this size, outgrowth of clones which can definitively escape from $\mathrm{T}$ cell lyse, appears to be inevitable. It is also known that these $\mathrm{T}$ cell resistant clones exhibit genetic dysregulation-related irreversible and unrepairable quantitative and qualitative alterations in their MHC-I antigen production. It results in a growing inability of the presentation of tumor associated antigens for the cytotoxic T cells [4-
6]. Because of this vulnerability of MHC-I restricted adaptive immune responses growing attention is focusing on the MHC-I unrestricted innate immune mechanisms whose escape mechanisms appear to be more repairable. As it's well known the MHC-I unrestricted effector cells have regularly a basic activity (so called priming) which can determine their functions. Similar to neuroendocrine system this priming of innate immune system exhibits also a polarity. Namely, it is committed in two directions. As shown in Figure 1 the type- 1 macrophages (M1) and from monocytes originated type-1 dentritic cells (D1) generate proinflammatory cytokines (only at short time), facilitate the production of IL-12, activate cytotoxic effectors, such

*Correspondence to: Hajto T, MD, PhD. Meggyes u. 26, H-2045 Törökbálint, Hungary, Tel: +36 309735 337; E-mail: drhajtot@t-online.hu

Key words: Pancreas carcinoma, Gemcitabine, Nab-paclitaxel, Immunomodulatory treatment, Stress-related molecules, Rice bran arabinoxylan concentrate, MGN-3/ Biobran

Received: Novemer 24, 2020; Accepted: Novemer 30, 2020; Published: December 03, 2020 


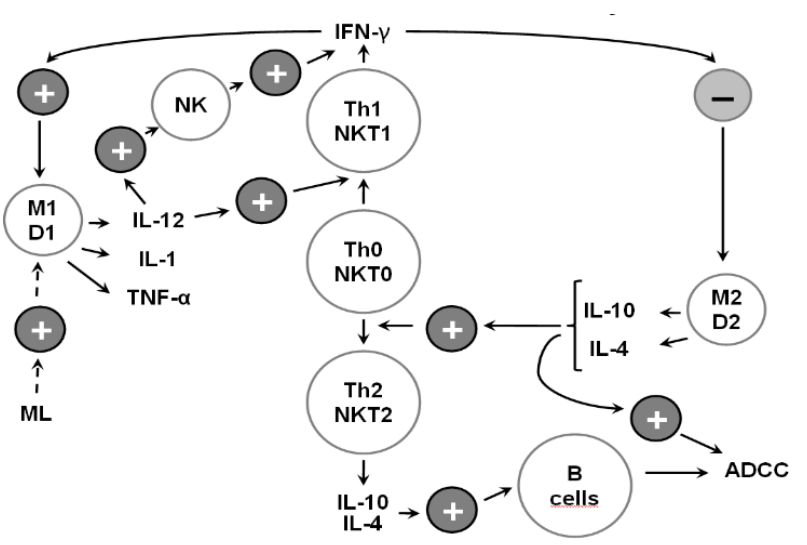

Figure 1. The innate immune system is committed in two directions; M1 and D1 are type-1 macrophages and dentritic cells which take part in the regulation the antitumor killer cells. M2 and D2 are type-2 macrophages and dentritic cells which facilitate the generation of Th2 cells and inhibit the type-1 system. Moderately modificated Illustration from JS. Murray

as natural killer (NK), gamma-delta T and type-1 NKT1 cells which are potent inhibitors of tumor growth in MHC unrestricted manner [7-9]. However, these type-1 innate immune cells are down regulated in tumor disease [9]. Moreover, available information suggests that there is a tumor-induced dominance of type-2 macrophages (M2) and of the from the plasmocytoid precursors originated type-2 dentritic (D2) cells which generate IL-4 and IL-10 facilitating the generation of Th2 cells and inhibiting the type- 1 system. It was also shown that M2 and D2 cells affect chronic inflammation, promote cell proliferation by producing growth factors and stimulate the angiogenesis. Parallel with the down regulation of type- 1 cells, it was also found that tumor patients can have up to $40 \%$ type- 2 peripheral monocytes in contrast to healthy persons who have only $10 \%[7,8]$.

In the immunological research always more data are discovered which support this polarity and in the cases of pancreas tumor the dominance of type- 2 mechanisms appear to play a considerable role. It has been repeatedly shown that the peripheral level and cytotoxic activity of NK cells are positively correlated with survival of PDAC patients $[1,2]$. New evidences indicate that PDAC cells release metabolites favoring the expansion and accumulation of monocyticmyeloid derived suppressor cells resulting in an increased levels of both circulating and tumor infiltrating type- 2 innate immune cells and this disturbed balance is associated with the shorter overall survival of PDAC [3].

Gemcitabine with nab-paclitaxel as a standard first line therapy is reporting improved clinical parameters but it is only transient. More than 15 year ago it was suggested that Gemcitabine in spite of its decreasing effect on peripheral level of lymphocytes appears to be less immunosuppressive compared to other cytostatic drugs [10]. In the background of these interesting observations a new discovery of Japan investigators may give now at least in part an explanation. Gemcitabine can increase the sensitivity of tumor cells to NK cytotoxicity enhancing the expression of their ligands such as MHC class-I chain-related A and $\mathrm{B}$ (MICA and MICB) molecules [11].

Consequently, this case report may help to support the hypothesis that the effect of gemcitabine will be increased if it will combined with a type- 1 innate immune cells activating evidence based and standardized immunomodulator (arabinoxylan concentrate (MGN-3/Biobran).

\section{Material and Methods}

\section{Combination of gemcitabine plus nab-paclitaxel with standardized rice bran extract (BioBran/MGN-3)}

The immunomodulatory drug used in the combinative treatment of the presented patient is BioBran/MGN-3 which is manufactured and supplied by Daiwa Pharmaceutical Co, Ltd, Tokyo, Japan. BioBran/ MGN-3 is composed of denaturated hemicellulose, which is obtained from rice bran hemicellulose reacting with multiple carbohydratehydrolyzing enzyme from shiitake mushrooms. BioBran/MGN-3 is standardized for its main chemical component: arabinoxylan with a xylose (in its main chain) and with an arabinose polymer (in its side chain). To the presented patient BioBran/MGN-3 was given orally in doses of $45 \mathrm{mg} / \mathrm{kg}$ three times a week and it was combined with eight cycles gemcitabine $(1678 \mathrm{mg})$ and nab-paclitaxel $(210 \mathrm{mg})$ on 1.- 8. - 15. days of months.

\section{Ethics committee}

Ethics committee proposed to observe and publish case reports of own patients treated with standardized plant immunomodulators. The patient has given an informed consent to process and publish her dates. This case report may stimulate an interest for other research groups according to the opinion of the ethics committee.

\section{Results}

In a now 56 years old patient in January 2020 in caudal part of pancreas an inoperable ductal adenocarcinoma (39x46 mm) with multiplex liver metastases $(10-30 \mathrm{~mm})$ was established by CT and biopsy. His tumor markers were elevated: CA-19-9 was higher than $10000 \mathrm{U} / \mathrm{ml}$ and the carcino-embryional antigen (CEA) level was $26.76 \mathrm{ng} / \mathrm{ml}$. He had a deteriorating quality of life. He was in a nearly terminal state (having $27 \mathrm{~kg}$ decrease in body weight and intensive pains). From February 2020 until November 2020 he was treated with eight cycles gemcitabine $(1678 \mathrm{mg})$ and nab-paclitaxel $(210 \mathrm{mg})$ on 1.8. - 15. days of months. This therapy was regularly combined with 45 mg / kg Biobran /MGN-3 given per oral three times a week. In April 2020 a remission of pancreas tumor $(25 \times 38 \mathrm{~mm})$ and average $3-10$ $\mathrm{mm}$ decreases in liver metastases were established by CT. At this time tumor markers are also decreased: CA 19-9 was $1172 \mathrm{U} / \mathrm{ml}$ and CEA $3.0 \mathrm{ng} / \mathrm{ml}$. In August 2020 a complete remission of pancreas tumor was found and the liver metastases showed further $3-6 \mathrm{~mm}$ decreases. The tumor marker were normalized (CA 19-9 was $22 \mathrm{U} / \mathrm{ml}$ and CEA was $2.0 \mathrm{ng} / \mathrm{ml}$. Figures 2 and 3 demonstrate these results monitoring the tumor diameters and the CA 19-9 tumor marker. These remissions were in November 2020 also established. CA-19-9 was $14.5 \mathrm{U} / \mathrm{ml}$. The patient has (after a therapy for 10 months) at the end of November 2020 no. any complaints and can work $100 \%$.

\section{Discussion}

As it known the overall survival (OS) of metastatic pancreatic ductal adenocarcinoma patients is very seldom longer than one year. Gemcitabine with nab-paclitaxel as a standard first line treatment is reporting improved median OS of 8.7 months compared with 6.6 months for gemcitabine alone [12-14]. Other authors did not found this difference in OS, but the progression free survival and the objective response rate were found to be improved [15]. There is now a general clinical opinion that in the treatment of metastatic PDAC with advanced stages of disease each sort of chemotherapy offers only very modest gain, 
Hajto T (2020) Can standardized and evidence based plant immunomodulator (rice bran arabinoxylan concentrate) increase the effect of Gemcitabine in a synergistic manner? Case report of a patient with ductal pancreas carcinoma

\section{The longest diameter of pancreas tumor}

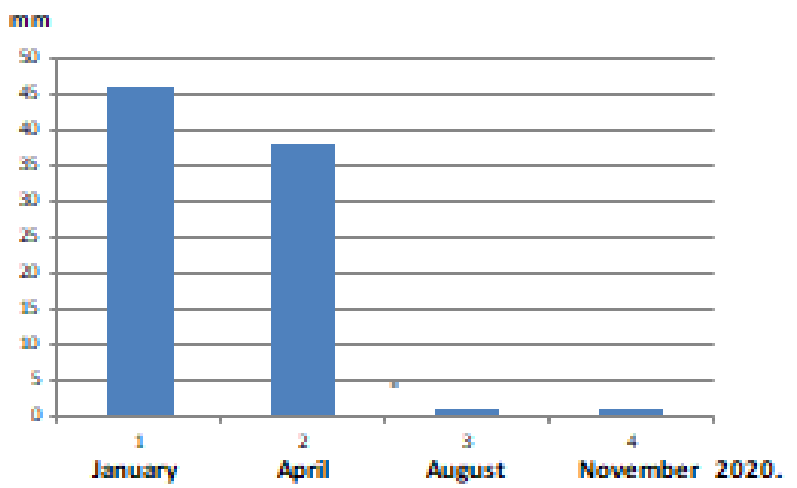

Figure 2. Momitoring the greatest tumor diameters of a patient with pancreas ductal adenocarcinoma; Patient with PDAC were treated with eight cycles of gemcitabine and nab-paclitaxel combined with an evidence based immunomodulator (standardized rice bran arabinoxylan concentrate (MGN-3 / Biobran)

\section{CA 19-9 values of patient with ductal pancreas carcinoma \\ $\mathrm{u} / \mathrm{ml}$}

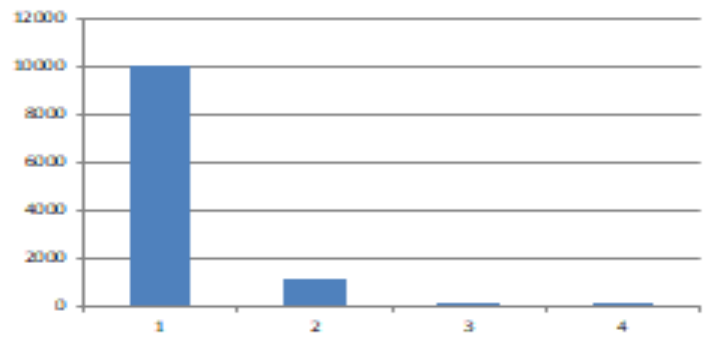

January April August November 2020.

Figure 3. Monitoring of tumor marker of patient with PDAC; Patient with PDAC were treated with eight cycles of gemcitabine and nab-paclitaxel combined with an evidence based immunomodulator (standardized rice bran arabinoxylan concentrate (MGN-3 / Biobran)
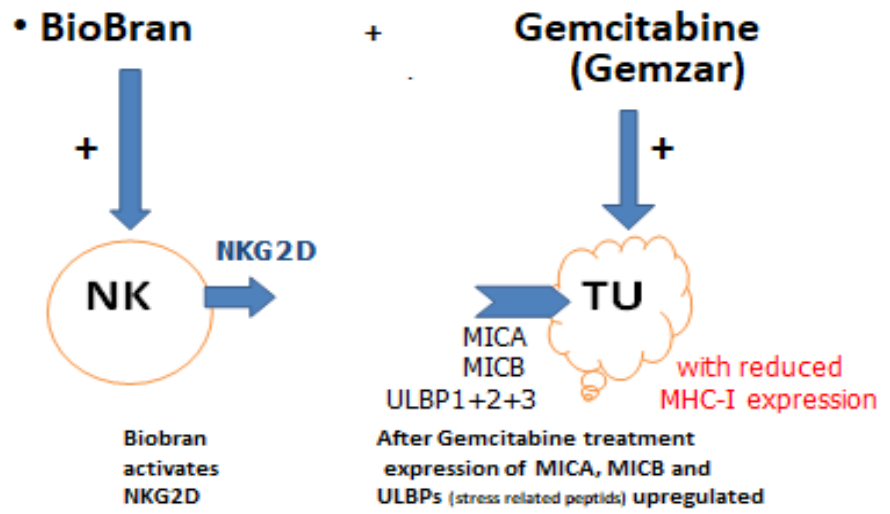

Figure 4. Combination of gemcitabine with an evidence based immunomodulator such as arabinoxylan concentrate (MGN-3/Biobran); The combination of gemcitabine with a standardized rice bran concentrate (Biobran) is therefore promising since gemcitabine enhances the NK sensitivity of tumor cells upregulating the stress related molecules (MICA and B) on cell surface of tumor cells which are the ligands of the most important killing activator receptors (NKG2D) on NK cells. If the NK cells are parallel also activated by Biobran /MGN-3 it may result's in a synergistic effect
Presented case report may support an old hypothesis that immunotherapy can increase the effect of gemcitabine [10]. Therefore the results of Morisaki, et al. are very exciting [11]. These authors investigated the effect of Gemcitabin on MICA and MICB expression in culture of T24 urothelial cancer cells. Gemcitabine given in 0.1 microgram $/ \mathrm{ml}$ concentration was able to induce a more than twice increase in expression of these stress-related molecules on cancer cells. Interestingly higher doses (for example 1 microgram / ml) were less effective. Other authors have also established these results [12]. It was also shown that in presence of gemcitabine the NK sensitivity of T24 urothelial cancer cells with and without IL-2 was also elevated resulting in significant elevated NK function [11]. Present clinical case report can support these in vitro results and it is also in agreement with previous clinical case reports [9]. Figure 4 represents the possible explanation of the synergestic effect between gemcitabine and immunomodulator (MGN-3). This combination appears therefore to be important since gemcitabine given alone was found to diminish the NK activity because of its decreasing effect on IFN-gamma production which is necessary at least in part for the maintenance of the type-1 immune cell activity [2].

Pathogenic Associated Molecular Pattern (PAMP) molecules are the best candidates for a type- 1 effectors activating immunomodulation. However, PAMP molecules exist only in the nature (bacteria or plants). The chemistry is not able to produce these configurations. Bacteria have toxic side effects but plants not. Unfortunately, the best evidence based and standardized immunodulators without any side effects are plant extracts and they are all over the world registered only as food supplements independent on that as to whether they are evidence based or not. There are growing evidences that plant PAMP-like molecules (such as standardized rice bran arabinoxylan concentrate) exhibit a lot of immunological [16-27] and clinical benefit [28-30]. For example, a randomized double blind clinical trial showed that the combination of arabinoxylan concentrate with chemotherapy enhanced six times the two years survival of liver cancer patients [28].

The research of evidence based immunomodulators has therefore a great clinical interest since protein kinase signaling pathway inhibitors such as Mitogen activated Extracellular signal regulation Kinase (MEK) can also enhance the expression of stress related molecules similar to gemcitabine [9,31]. It appears that growth factors stimulate the cell proliferation on the one hand and protect the cells against immune effectors (down regulating their stress related ligands). Consequently, similar to gemcitabine a combination of protein kinase signaling pathway inhibitors and the type- 1 natural effector cells activating immunomodulators may also open new perspectives in the tumor therapy.

\section{Disclosure Statement}

The authors declare that there is no competing or other conflicting interest in relation to this paper.

\section{References}

1. Adamska A (2017) Pancreas ductal adenocarcinoma: current and evolving therapy. Int J Mol Sci 18: 1336. [Crossref]

2. Marherie D (2012) Effect of pemetrexed on innate immune killer and adaptive T cells in subjects with adenocarcinoma of the pancreas. J Immunother 35: 629-640. [Crossref]

3. Trovato R (2019) Immunosuppression by monocytic-myeloid derived suppressor cells in patients with pancreas ductal carcinoma is orchestrated by STAT3. J Immunother 7: 255. [Crossref]

4. Seliger B, Maeurer MJ, Ferrone S (2000) Antigen-processing machinery breakdown and tumor growth. Immunol Today 21: 455-464. [Crossref] 
Hajto T (2020) Can standardized and evidence based plant immunomodulator (rice bran arabinoxylan concentrate) increase the effect of Gemcitabine in a synergistic manner? Case report of a patient with ductal pancreas carcinoma

5. Doan T, Melvold R, Viselli S, Waltenbaugh C (2008) Presentation by MHC class I. In: Harvey RA, Champe PC (eds): Immunology, Philadelphia, Lippincott Williams and Wilkins, pp: 123-126.

6. Male D, Brostoff J, Roth DB, Roitt I (2013) Immunity to Cancer. In: Hyde M, and Vosburgh A (eds): Immunology. International Edition, Elsevier, pp: 355-365.

7. Sánchez-Torres C, García-Romo GS, Cornejo-Cortés MA, Rivas-Carvalho A, SánchezSchmitz G (2001) CD16 ${ }^{+}$and CD16- human blood monocyte subsets differentiate in vitro to dentritic cells with different abilities to stimulate $\mathrm{CD}^{4+} \mathrm{T}$ cells. Int Immunol 13: 1571-1581. [Crossref]

8. Mantovani A (2007) Inflammation and cancer; the macrophage connection. Medicina (Buenos Aires) 67: 32-34

9. Hajto $\mathrm{T}$ (2018) New perspectives to improve the MHC-I unrestricted immune mechanisms against malignant tumors. Adv Clin Trans Res 2: 1-13.

10. Plate JMD (2005) Effect of Gemcitabine on immune cells in subjects with adenocarcinoma of the pancreas. Cancer Immunol Immunother 54: 915-925. [Crossref]

11. Morisaki T, Hirano T, Koya N (2014) NKG2D-directed cytokine-activated killer lymphocyte therapy combined with gemcitabine for patients with chemoresistant metastatic solid tumors. Anticancer Res 34: 5227. [Crossref]

12. Okita R, Wolf D, Yasuda K (2015) Contrasting effects of cytotoxic anticancer drug Gemcitabine and the EGFR tyrosine kinase inhibitor Gefitinib on NK cell-mediated cytotoxicity via regulation of NKG2D ligand in small-cell lung cancer cells. PloS One 10: 0139809. [Crossref]

13. Xie X, Zhou Y, Wang X (2017) Enhanced antitumor activity of gemcitabine by polysaccharide-induced NK cell acivation. Carbohydrate Polym 173:360-371. [Crossref]

14. Von Hoff DD (2013) Increased survival in pancreatic cancer with nab-paclitaxel plus gemcitabine. N Engl J Med 369: 1691-1703.

15. Carrie PG (2020) Scheduling nab-paclitaxel combind with gemcitabine. British $J$ Cancer 122: 1760-1768. [Crossref]

16. Cholujova D, Jakubikova J, Czako B, Martisova M, Hunakova L, et al. (2012) MGN3 arabinoxylan rice bran modulates innate immunity in multiple myeloma patients. Cancer Immunol Immunother 62: 437-445. [Crossref]

17. Cholujova D, Jakubikova J, Sedlak J (2009) BioBran-augmented maturation of human monocyte-derived dendritic cells. Neoplasma 56: 89-95. [Crossref]

18. Tan BL, Norhaizan ME (2017) Scientific Evidence of Rice By-Products for Cancer Prevention: Chemopreventive Properties of Waste Products from Rice Milling on Carcinogenesis In Vitro and In Vivo. Biomed Res Int 2017: 9017902. [Crossref]

19. Pérez-Martínez A, Valentín J, Fernández L, Hernández-Jiménez E, López-Collazo E, et al. (2015) Arabinoxylan rice bran (MGN-3/Biobran) enhances natural killer cell-mediated cytotoxicity against neuroblastoma in vitro and in vivo. Cytotherapy 17: 601-612. [Crossref]
20. Badr El-Din NK, Ali DA, Alaa El-Dein M, Ghoneum M (2016) Enhancing the Apoptotic Effect of a Low Dose of Paclitaxel on Tumor Cells in Mice by Arabinoxylan Rice Bran (MGN-3/Biobran). Nutr Cancer 68: 1010-1020. [Crossref]

21. Badr El-Din NK, Abdel Fattah SM, Pan D, Tolentino L, Ghoneum M (2016) Chemopreventive Activity of MGN-3/Biobran Against Chemical Induction of Glandular Stomach Carcinogenesis in Rats and Its Apoptotic Effect in Gastric Cancer Cells. Integr Cancer Ther 15: 26-34. [Crossref]

22. Ghoneum M, Agrawal S (2014) Mgn-3/biobran enhances generation of cytotoxic CD8+ $\mathrm{T}$ cells via upregulation of dec-205 expression on dendritic cells. Int J Immunopathol Pharmacol 27: 523-530. [Crossref]

23. Ghoneum M, Badr El-Din NK, Ali DA, El-Dein MA (2014) Modified arabinoxylan from rice bran, MGN-3/biobran, sensitizes metastatic breast cancer cells to paclitaxel in vitro. Anticancer Res 34: 81-87. [Crossref]

24. Ghoneum M, Badr El-Din NK, Abdel Fattah SM, Tolentino L (2013) Arabinoxylan rice bran (MGN-3/Biobran) provides protection against whole-body $\gamma$-irradiation in mice via restoration of hematopoietic tissues. J Radiat Res 54: 419-429. [Crossref]

25. Ghoneum M, Agrawal S (2011) Activation of human monocyte-derived dendritic cells in vitro by the biological response modifier arabinoxylan rice bran (MGN-3/Biobran). Int J Immunopathol Pharmacol 24: 941-948. [Crossref]

26. Badr El-Din NK, Noaman E, Ghoneum (2008) In vivo tumor inhibitory effects of nutritional rice bran supplement MGN-3/Biobran on Ehrlich carcinoma-bearing mice. Nutr Cancer 60: 235-244. [Crossref]

27. Gollapudi S, Ghoneum M (2008) MGN-3/Biobran, modified arabinoxylan from rice bran, sensitizes human breast cancer cells to chemotherapeutic agent, daunorubicin Cancer Detect Prev 32: 1-6. [Crossref]

28. Bang MH, Riep TV, Thinh NT, Song LH, Dung TT, et al. (2010)Arabinoxylan rice bran (MGN-3) enhances the effect of interventional therapies for the treatment of hepatocellular carcinoma: A three-year rabdomized clinical trial. Anicancer Res 30: 5145-5152. [Crossref]

29. Itoh Y, Mizuno M, Ikeda M, Nakahara R, Kubota S, et al. (2015) A Randomized, Double-Blind Pilot Trial of Hydrolyzed Rice Bran versus Placebo for Radioprotective Effect on Acute Gastroenteritis Secondary to Chemoradiotherapy in Patients with Cervical Cancer. Evid Based Complement Alternat Med 2015: 974390. [Crossref]

30. McDermott C, Richards SC, Thomas PW, Montgomery J, Lewith G (2006) A placebocontrolled, double-blind, randomized controlled trial of a natural killer cell stimulant (BioBran MGN-3) in chronic fatigue syndrome. QJM 99: 461-468. [Crossref]

31. Bae JH, Kim SJ, Kim MJ (2012) Sussceptibility to natural killer cell-mediated lysis of colon cancer cells is enhanced by treatment with epidermal growth factor receptor inhibitors through UL16 binding protein-1 induction. Cancer Sci 103 : 7-16. [Crossref]

Copyright: (C2020 Hajto T. This is an open-access article distributed under the terms of the Creative Commons Attribution License, which permits unrestricted use, distribution, and reproduction in any medium, provided the original author and source are credited. 